\title{
The late positive potential as a marker of motivated attention to underweight bodies in girls with anorexia nervosa
}

\author{
Stefanie Horndasch ${ }^{\mathrm{a}, *}$, Hartmut Heinrich ${ }^{\mathrm{a}, \mathrm{b}}$, Oliver Kratz ${ }^{\mathrm{a}}$, Gunther H. Moll ${ }^{\text {a }}$ \\ a Department of Child and Adolescent Mental Health, University of Erlangen-Nuremberg, Schwabachanlage 6 / 10, 91054 Erlangen, Germany \\ ${ }^{\text {b } H e c k s c h e r ~ K l i n i k u m, ~ D e i s e n h o f e n e r ~ S t r a ß e ~ 28, ~} 81539$ Munich, Germany
}

\section{A R T I C L E I N F O}

\section{Article history:}

Received 16 March 2012

Received in revised form 27 September 2012

Accepted 29 September 2012

\section{Keywords:}

Anorexia nervosa

Adolescents

Late positive potential

Event related potentials

Motivation

\begin{abstract}
A B S T R A C T
Objective: In anorexia nervosa (AN), aspects of motivational salience and reward are increasingly discussed. Event related potentials, particularly the late positive potential (LPP), have been investigated as a marker for motivational salience of stimuli, for example in addictive disorders. The aim of this study was to assess the LPP as a possible indicator of motivated attention towards disease-specific pictures of underweight female bodies in adolescents with AN in comparison to typically developing (TD) adolescent girls.

Method: 13 girls with AN and 18 TD adolescent girls (aged 12 to 18 years) viewed pictures of underweight, normal-weight and overweight women while EEG activity was recorded. An earlier (450-680 ms after stimulus onset) as well as a later time window (850-1250 ms after stimulus onset) of the LPP were examined for the different picture categories. Participants were also asked to rate subjective emotions (fear, disgust, happiness) elicited by the pictures.

Results: Subjective ratings showed no differential experience of emotions for the two groups. For AN patients, highest LPP amplitudes were found for underweight women in the earlier as well as in the later time window. In TD girls, highest amplitudes for pictures of overweight women were observed in the earlier time window.

Conclusion: A differential LPP pattern for girls with AN and TD girls when viewing pictures of women's bodies of different weight categories was obtained. Highest amplitudes in AN patients for pictures of underweight women may reflect motivational significance of strongly underweight body shapes.
\end{abstract}

(c) 2012 Elsevier Inc. All rights reserved.

\section{Introduction}

Anorexia nervosa (AN), with a prevalence of $0.5-1 \%$ in adolescents and young adults, has highest incidence rates for females aged 15-19 [1]. Patients with AN overevaluate their shape and weight [2] and develop organized cognitive structures around the issue of weight and its implications for the self [3]. In that context, an important role of motivational salience is increasingly discussed: Altered dopamine function in general and also with a focus on the role of the mesolimbic reward system of the brain (with the ventral striatum as a core structure) has been evaluated [4]. In an fMRI study Fladung et al. observed differential activation patterns in response to underweight, normal-weight and overweight computer-generated body stimuli. Asked to view the stimuli in a self-referring way, AN patients showed higher activation in the ventral striatum while viewing underweight compared with normal-weight stimuli whereas in healthy women a reverse pattern was demonstrated [5]. The authors interpret these findings as reward reactions in line with theories of

\footnotetext{
* Corresponding author. Tel.: + 499131 8539123; fax: + 4991318539126. E-mail address: stefanie.horndasch@uk-erlangen.de (S. Horndasch).
}

starvation dependence accounting for the maintenance of AN [6] or eating disorders as a form of addiction [6,7].

Examining neural correlates of body dissatisfaction Friederich et al. found increased activation of the insula and premotor cortex (regions which in some other studies were shown to be involved in interoceptive awareness and self-body representation) in AN patients when comparing themselves to slim fashion models and see their findings associated with implicit motivation towards disease-related cues [8].

Whereas evidence supporting this approach relies on neuropsychological, neurobiological and brain imaging studies, an investigation on central nervous reactions towards disease-specific stimuli using event related potentials (ERPs) has not yet been reported.

However, ERPs may provide several advantages in the study of stimulus processing, especially when concerned with aspects of motivational salience. They have been shown to be a specific indicator of the selective visual processing and also of motivational relevance of stimuli. The activation of distinct brain areas shown in fMRI studies on the one hand tends to leave room for different interpretations as various neural regions are generally associated with a wide variety of phenomena and has recently often been criticized as not specific enough to detect discrete emotional states [9]. On the other hand 
fMRI imaging is not able to provide information on distinct early attentional processes because of only low temporal resolution.

ERPs are stimulus- and time-locked changes in the ongoing EEG which allow the study of covert neural processing with high temporal resolution [10]. A relatively late, sustained positive ERP is referred to as the late positive potential (LPP). The LPP is larger following the presentation of both pleasant and unpleasant compared to neutral and largest for highly arousing stimuli $[11,12]$. Emotional modulation of the LPP has been found over centro-parietal regions [13,14] with a shift from a parietal positivity in the $400-1000 \mathrm{~ms}$ time range to a more broadly superior positivity in the $1000-2000 \mathrm{~ms}$ range following stimulus onset [15]. Whereas earlier LPP components are linked with capture of motivated attention, the later LPP may reflect sustained attentive processing of motivationally salient stimuli related to ongoing evaluations of stimulus meaning [13].

The LPP has also been studied in the context of substance abuse and addiction: Increased attention towards substance-related stimuli, elicited by the activation of motivational systems, is reflected by larger LPP amplitudes e.g. in cocaine-dependent patients [16], alcohol drinkers [17] and smokers [18] with an association between drug craving and ERP amplitudes.

We aimed to assess the LPP as a possible marker for motivational significance of disease-specific pictures of female bodies in adolescents with AN in comparison to typically developing adolescent girls. We were interested in the time course of neural activity and subjective emotional responses to different body schemes (underweight, normalweight and overweight). In line with the observations made in patients with addictive disorders and imaging studies showing differential neural activation towards disease-specific stimuli in AN we expected enlarged LPP amplitudes as a marker for motivated attention towards pictures of underweight women in patients.

\section{Method}

\section{Participants}

We included 13 female adolescent patients fulfilling the criteria for anorexia nervosa (ICD-10 F50.0), diagnosed by an experienced child psychiatrist or psychologist. The sample comprised inpatients and outpatients treated with a cognitive-behavioral therapy program. Two patients with anorexia nervosa had a comorbid major depressive disorder and received antidepressant medication (SSRI). Exclusion criteria were current or a history of psychiatric disorder (TD girls), medication with narcotics and severe somatic, including neurological disease.

Control subjects were 18 TD adolescent girls recruited from local high schools with a BMI within the normal range of the respective age percentiles.

Of originally 38 participants seven participants had to be excluded because of insufficient EEG data quality. They were all control participants and were comparable to the rest of the control group concerning BMI age percentile, EDI total score and all EDI subscales (all $\mathrm{p}>.28$, $\mathrm{t}(23)<1.0)$.

Written informed consent was obtained from all participants and their parents. The study was conducted in accordance with the Declaration of Helsinki and was approved by the Ethics Committee of the University Clinic Erlangen.

\section{Procedure}

First the participants filled out a German version of the Eating Disorder Inventory-2 [19] and a psychometric interview was conducted to assess exclusion criteria. For viewing the pictures, the participants were seated comfortably at a distance of $60 \mathrm{~cm}$ from a computer monitor. The order of the pictures was randomly assigned to control for potential order effects. Each picture was presented for $8 \mathrm{~s}$. After viewing each picture participants were asked to rate their experience of different emotions (fear, disgust and happiness) on a custom-made rating scale of 0 (not at all) to 5 (very high) to get information on the extent to which those basic emotions that seemed most relevant to the study context were experienced. Emotion categories fear and disgust were preferred over a simple rating of negative and positive affect to differentiate more clearly possible ratings of aversion. Total duration of the experiment was approximately $30 \mathrm{~min}$. Finally, the participants' height and weight were measured.

\section{Stimulus material}

Full body pictures of three different views (front, rear, profile) of women in either a bathing suit or undergarment were used, so that the majority of the body was unclothed. The pictures were allocated to three different body schemes (underweight, normal-weight and overweight, 5 pictures each) according to a rating by 8 health professionals. The pictures were displayed in black and white and without showing the head of the person to control for effects of picture luminance or brightness and facial expression. The number of images per weight category was rather low so that the signal-to-noise ratio of the averaged EPs was also low. So, in our opinion, this indicates rather a robust effect than an artificially induced effect. On the other hand, habituation effects could occur if a larger number of stimuli per category was used.

\section{Data acquisition}

EEG activity was recorded according to the 10-20 international system, amplified, and digitized at a sampling rate of $500 \mathrm{~Hz}$ using an active amplifier system (Brain Products, Germany). The recordings were referenced to $\mathrm{Cz}$ and re-referenced offline to linked mastoids. Horizontal and vertical eye movements were recorded.

EEG was filtered $(0.05 \mathrm{~Hz}-20 \mathrm{~Hz})$ and blinks were corrected using the Gratton and Coles algorithm. Trials contaminated by artifacts were eliminated.

After baseline correction (100 ms pre-stimulus as baseline), average ERP waves were calculated for separate stimulus categories (see Fig. 1). To study the time resolution in more detail, we divided the LPP into two time windows (earlier LPP: 450-680 ms, later LPP: 850-1250 ms after stimulus onset). Topographic analysis showed maximal amplitudes of the earlier component at parietal and of the later component at central recording sites.

\section{Statistics}

For group comparisons concerning clinical variables (age, BMI, BMI age percentile, EDI-2 total score and subscores) t-tests were conducted. Concerning the emotion ratings, an analysis of variance (ANOVA) with the within-subject factor category (3, underweight, normal-weight, overweight) and the between-subject factor group (2, AN and TD) was conducted for each of the different emotions being rated (fear, disgust, happiness).

Mean brain electrical activity of the earlier (450-680 ms) and later (850-1250 ms) LPP time window were used as measures of stimulus processing and subjected to an ANOVA with the within-subject factors category (3, underweight, normal-weight, overweight), electrode (3, P3, Pz and P4 in the earlier time window and C3, Cz and C4 in the later time window, respectively) and the between-subject factor group (2, AN and TD). For significant group $\times$ category interactions, it was planned to compute trend scores (linear, quadratic) for each group separately, which allowed studying the relation of the different weight categories. Greenhouse-Geisser correction was used when appropriate. We also tested for correlations between emotion ratings and LPP amplitudes by calculating a difference score between reactions (ratings and LPP amplitudes: earlier component, averaged over the 

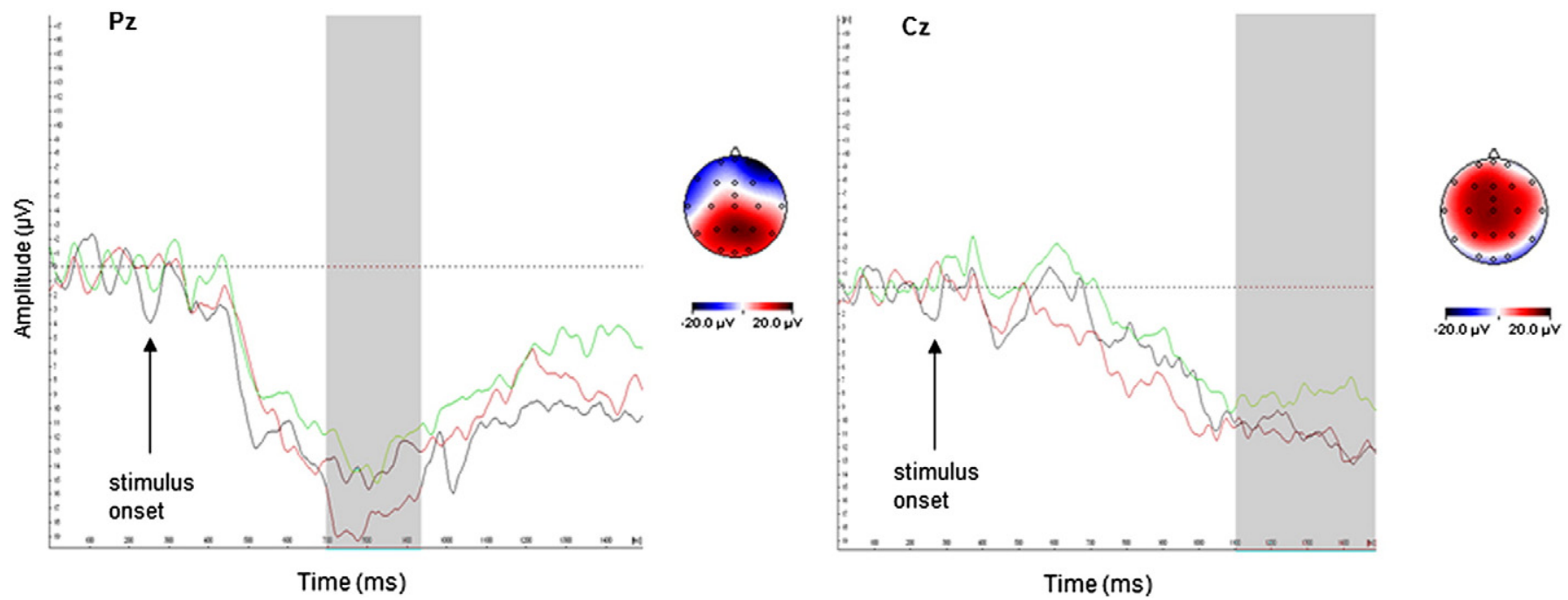

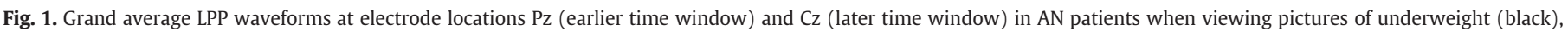

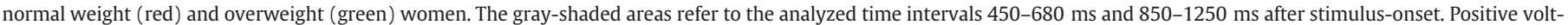
ages are plotted downwards as per convention. The topographic map shows maximum LPP at parietal recording sites.

parietal electrodes, and later component, averaged over the central electrodes) towards picture categories underweight and normal weight (Fig. 2).

Mean values \pm standard deviations are reported.

\section{Results}

Participant characteristics

AN patients and TD participants were of similar age (AN 15.7 \pm 1.8 , TD $16.6 \pm$ 1.8 years) but differed significantly on BMI and BMI age percentiles. On the EDI-2, the AN group reported significantly greater total scores and values on most of the subscales (see Table 1 ).

\section{Emotion ratings}

For the values of the experienced emotions fear, disgust and happiness a highly significant effect for weight category (for all emotions $\mathrm{p}<.001$ ) was found. Both groups (AN and TD) reported more fear and disgust when looking at pictures of under- and overweight women than at those of normal-weight women and more happiness when looking at normal-weight than at under- and overweight women. No significant group or group $\times$ category effect was found (see Table 2 ).

Earlier LPP component (450-680 ms after stimulus onset)

A significant main effect of electrode was obtained $(F(2,58)=4.3, p<.02)$ indicating greatest amplitudes over the midline electrode $(\mathrm{Pz})$. A significant interaction category $\times$ group was observed $(F(2,58)=5.1, p<.01)$. Group-specific analysis revealed

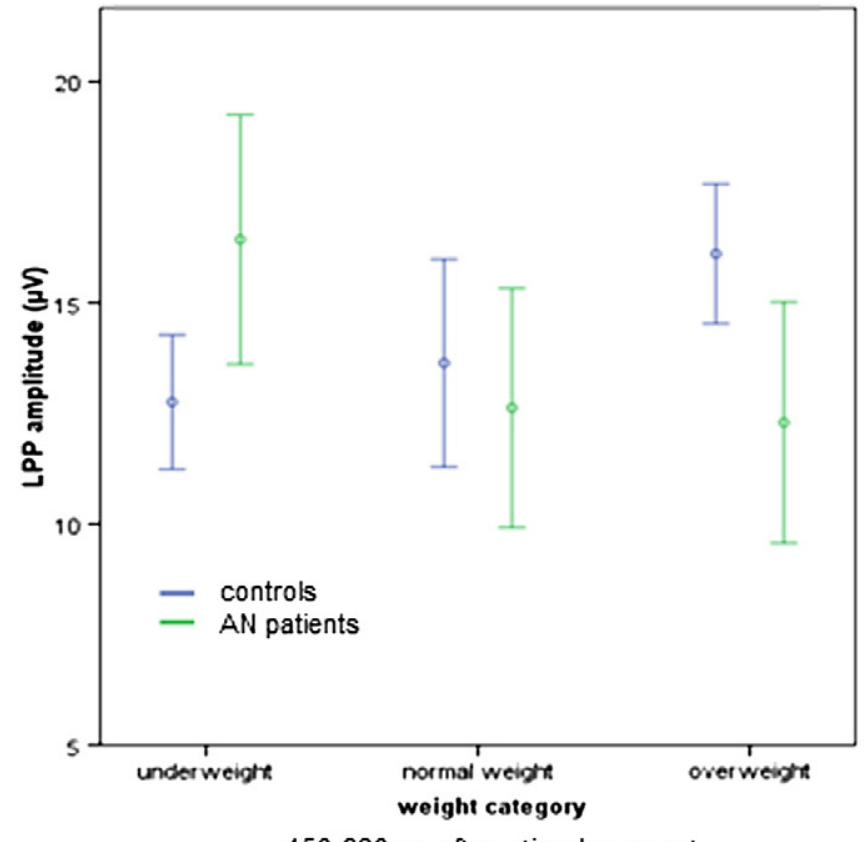

$450-680 \mathrm{~ms}$ after stimulus onset



$850-1250 \mathrm{~ms}$ after stimulus onset

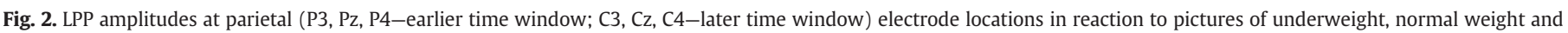
overweight women (mean \pm standard error). 
Table 1

Sample characteristics.

\begin{tabular}{lccl}
\hline & $\begin{array}{l}\text { Patients } \\
\text { with AN } \\
(\mathrm{n}=13)\end{array}$ & $\begin{array}{l}\text { Typically } \\
\text { developing } \\
\text { girls }(\mathrm{n}=18)\end{array}$ & $\begin{array}{l}\text { Statistics } \\
(\mathrm{t} \text {-tests })\end{array}$ \\
\hline Age (years) & $15.7 \pm 1.8$ & $16.6 \pm 1.8$ & $\mathrm{t}(29)=-1.4, \mathrm{n} . \mathrm{s}$. \\
BMI $\left(\mathrm{kg} / \mathrm{m}^{2}\right)$ & $17.5 \pm 1.1$ & $21.7 \pm 1.6$ & $\mathrm{t}(29)=-8.4, \mathrm{p}<.001$ \\
BMI age percentile & $13.2 \pm 8.9$ & $65.7 \pm 26.5$ & $\mathrm{t}(29)=-6.8, \mathrm{p}<.001$ \\
& & & \\
Eating Disorder Inventory $($ EDI-2) & $277.9 \pm 54.2$ & $199.5 \pm 36.9$ & $\mathrm{t}(29)=4.1, \mathrm{p}<.001$ \\
Total score & $16.0 \pm 6.9$ & $27.2 \pm 6.2$ & $\mathrm{t}(29)=4.7, \mathrm{p}<.001$ \\
Drive for thinness & $12.5 \pm 5.6$ & $11.1 \pm 4.1$ & $\mathrm{t}(29)=.9, \mathrm{n} . \mathrm{s}$. \\
Bulimia & $34.4 \pm 9.0$ & $26.6 \pm 7.3$ & $\mathrm{t}(29)=2.7, \mathrm{p}<.05$ \\
Body dissatisfaction & $30.3 \pm 9.8$ & $18.3 \pm 5.4$ & $\mathrm{t}(29)=4.4, \mathrm{p}<.001$ \\
Ineffectiveness & $20.0 \pm 5.9$ & $16.0 \pm 3.1$ & $\mathrm{t}(29)=2.5, \mathrm{p}<.05$ \\
Perfectionism & $22.5 \pm 6.5$ & $15.9 \pm 5.6$ & $\mathrm{t}(29)=3.0, \mathrm{p}<.01$ \\
Interpersonal distrust & $29.9 \pm 11.1$ & $19.7 \pm 7.1$ & $\mathrm{t}(29)=3.1, \mathrm{p}<.01$ \\
Interoceptive awareness & $26.3 \pm 4.2$ & $23.7 \pm 4.6$ & $\mathrm{t}(29)=1.6, \mathrm{n} . \mathrm{s}$. \\
Maturity fears & & & \\
\hline
\end{tabular}

in patients with AN a linear category effect $(\mathrm{T}-\operatorname{lin}(1,12)=5.1, \mathrm{p}<.05)$ with highest amplitudes when looking at pictures of underweight women $(16.4 \pm 10.2 \mu \mathrm{V})$, followed by those of normal-weight $(12.6 \pm 9.7 \mu \mathrm{V})$ and overweight $(12.3 \pm 9.8 \mu \mathrm{V})$ women. In TD girls, a linear effect of picture category $(\mathrm{T}-\operatorname{lin}(1,17)=6.1, \mathrm{p}<.05)$ was obtained indicating highest amplitudes when looking at pictures of overweight women $(16.0 \pm 6.4 \mu \mathrm{V})$, followed by picture categories normal-weight (13.8 \pm $10.1 \mu \mathrm{V})$ and underweight $(12.3 \pm 6.9 \mu \mathrm{V})$.

Later LPP component (850-1250 ms after stimulus onset)

A main effect of electrode $(F(2,58)=5.8, \mathrm{p}<.01)$ with highest amplitude at $\mathrm{Cz}$ was found. A significant interaction category $\times$ group $(F(2,58)=3.3, p<.05)$ was obtained. In AN patients, a significant category effect $(\mathrm{T}-\operatorname{lin}(1,12)=6,7, \mathrm{p}<.05)$ indicated greatest amplitudes for pictures of underweight women $(12.0 \pm 6.4 \mu \mathrm{V})$, followed by those of normal-weight $(9.0 \pm 7.1 \mu \mathrm{V})$ and overweight $(7.2 \pm 8.9 \mu \mathrm{V})$ women. In TD girls there was a tendency towards higher amplitudes for pictures of normal-weight women $(\mathrm{T}-$ quad $(1,17)=3.9, \mathrm{p}=.06)$.

Correlations between emotion ratings and LPP amplitudes

Differences between ratings of any emotion (fear, disgust and happiness) towards underweight and normal-weight women and LPP differences between amplitudes towards underweight and normal-weight women did not correlate $(|\mathrm{r}|<0.21, \mathrm{p}>.27)$.

\section{Discussion}

We aimed to investigate neural processing when viewing pictures of women's bodies in adolescent AN patients and TD adolescents with the help of the LPP, a marker of facilitated processing of and motivated attention towards stimuli. We found specific group differences: in an earlier as well as in a later time window LPP amplitudes of AN patients were highest when looking at pictures of underweight compared to normal-weight and overweight women. TD girls showed the highest amplitude in the picture category overweight in the earlier time window, maybe indicating a capture of attention by strongly overweight women at an earlier stage of stimulus processing, and a tendency

Table 2

Rating of emotions experienced when viewing pictures of the different weight categories (on a rating scale from 0 to 5 , mean \pm standard deviation).

\begin{tabular}{llll}
\hline Emotion & $\begin{array}{l}\text { Picture } \\
\text { category }\end{array}$ & $\begin{array}{l}\text { Patients } \\
\text { with AN }\end{array}$ & $\begin{array}{l}\text { Typically } \\
\text { developing girls }\end{array}$ \\
\hline Fear & Underweight & $1.3 \pm 1.2$ & $1.1 \pm 1.0$ \\
& Normal-weight & $0.3 \pm 0.6$ & $0.1 \pm 0.2$ \\
Disgust & Overweight & $0.7 \pm 1.2$ & $0.3 \pm 0.6$ \\
& Underweight & $1.4 \pm 1.3$ & $1.5 \pm 1.3$ \\
& Normal-weight & $0.2 \pm 0.4$ & $0.1 \pm 0.2$ \\
Happiness & Overweight & $1.8 \pm 1.4$ & $1.2 \pm 0.9$ \\
& Underweight & $0.1 \pm 0.2$ & $0.2 \pm 0.7$ \\
& Normal-weight & $1.1 \pm 1.0$ & $1.3 \pm 1.0$ \\
& Overweight & $0.1 \pm 0.2$ & $0.2 \pm 0.4$ \\
\hline
\end{tabular}

towards highest amplitudes in the category normal-weight in the later time window which hints towards sustained attentive and evaluative processing, maybe for the purpose of comparison.

The emotion ratings for the pictures in our study were comparable between patients and TD girls unlike in the study by Friederich et al. [8] where AN patients reported higher ratings of anxiety than control participants when confronted with slim models and also unlike in the study by Fladung et al. [5] when participants were asked to view body stimuli in a self-referring way ("feel-task" with group differences concerning different picture categories). However, in these tasks participants were explicitly asked to compare themselves to or imagine to actually look like the models; in our free viewing situation as in the "weight-task" in the study by Fladung et al., when participants were asked to assess the weight of the women, ratings did not differ between patients and controls. LPP amplitudes have been shown to be more pronounced for stimuli of positive and negative valence than for neutral ones with larger LPP amplitudes for pictures of nudes [12], so they leave open a variety of interpretations. But as participants of both groups in our study rated the emotional content of the pictures similarly and there were no significant correlations between LPP amplitudes and ratings in reaction to underweight in contrast to normal-weight women's pictures, the differential LPP pattern might not reflect basic emotional aspects of negative or positive valence as for example aversion or attraction, but primarily motivational salience and capture of attention.

In an fMRI study, a similar pattern related to the different weight categories had been found, i.e., an enlarged activation of the ventral striatum when looking at pictures of underweight women [5]. Another study [8] found increased activation of the insula as a sign of motivational significance of pictures of slim fashion models. Generally, pictures of thin women influence adolescents as widely accepted ideals for what their body should look like, but even more for patients with AN pictures of emaciated women (which serve as "thinspiration" on pro-anorexia websites) are common comparison targets and ideals and therefore highly motivating [20].

Although also stimuli of negative valence have been shown to elicit a pronounced LPP [11,12], as in our study might be the case concerning enlarged amplitudes for overweight women in TD girls, in line with ERP studies on craving reactions in drug dependency or misuse [16-18,21] the differential LPP effects for pictures of underweight women might support a model of AN as a phenomenon of increased pursuit of reward effects through self-starvation [6].

Possible limitations in the interpretation of the current results are the relatively small sample size, relatively high BMI of patients and heterogeneity of the sample with regard to treatment modality (outpatients and inpatients) and the small number of trials per category. However, the different patterns found for the two groups in both analyzed time windows hint towards the LPP as an indicator of motivational significance of pictures of underweight women.

\section{Competing interest statement}

The authors have no competing interests to report.

\section{Acknowledgments}

The authors thank Anna Holczinger, Stephanie Barth, Petra Studer, Thomas Högl and Martin Deinzer for their valuable support. Research was funded by the ELAN and IZKF programs of the University Erlangen-Nuremberg.

\section{References}

[1] Gowers S, Bryant-Waugh R. Management of child and adolescent eating disorders: the current evidence base and future directions. Journal of Child Psychology and Psychiatry Jan 2004;45:63-83.

[2] Fairburn CG, Harrison PJ. Eating disorders. Lancet Feb 1 2003;361:407-16. 
[3] Vitousek KB, Hollon SD. The investigation of schematic content and processing in eating disorders. Cognitive Therapy and Research 1990;14:191-214.

[4] Kaye W. Neurobiology of anorexia and bulimia nervosa. Physiology and Behavior Apr 22 2008;94:121-35.

[5] Fladung AK, Gron G, Grammer K, Herrnberger B, Schilly E, Grasteit S, et al. A neural signature of anorexia nervosa in the ventral striatal reward system. The American Journal of Psychiatry Feb 2010;167:206-12.

[6] Zink CF, Weinberger DR. Cracking the moody brain: the rewards of self starvation. Nature Medicine Dec 2010;16:1382-3.

[7] Davis C, Claridge G. The eating disorders as addiction: a psychobiological perspective. Addictive Behaviors Jul-Aug 1998;23:463-75.

[8] Friederich HC, Brooks S, Uher R, Campbell IC, Giampietro V, Brammer M, et al. Neural correlates of body dissatisfaction in anorexia nervosa. Neuropsychologia Aug 2010;48:2878-85.

[9] Lindquist KA, Wager TD, Kober H, Bliss-Moreau E, Barrett LF. The brain basis of emotion: a meta-analytic review. The Behavioral and Brain Sciences Jun 2012;35:121-43.

[10] Banaschewski T, Brandeis D. Annotation: what electrical brain activity tells us about brain function that other techniques cannot tell us-a child psychiatric perspective. Journal of Child Psychology and Psychiatry May 2007;48:415-35.

[11] Hajcak G, MacNamara A, Olvet DM. Event-related potentials, emotion, and emotion regulation: an integrative review. Developmental Neuropsychology Feb 12 2010;35:129-55.

[12] Schupp HT, Cuthbert BN, Bradley MM, Cacioppo JT, Ito T, Lang PJ. Affective picture processing: the late positive potential is modulated by motivational relevance. Psychophysiology Mar 2000;37:257-61.
[13] Schupp HT, Flaisch T, Stockburger J, Junghofer M. Emotion and attention: event-related brain potential studies. Progress in Brain Research 2006;156:31-51.

[14] Thiruchselvam R, Blechert J, Sheppes G, Rydstrom A, Gross JJ. The temporal dynamics of emotion regulation: an EEG study of distraction and reappraisal. Biological Psychology Feb 24 2011;87:84-92.

[15] Foti D, Hajcak G, Dien J. Differentiating neural responses to emotional pictures: evidence from temporal-spatial PCA. Psychophysiology May 2009;46:521-30.

[16] Franken IH, Dietvorst RC, Hesselmans M, Franzek EJ, van de Wetering BJ, Van Strien JW. Cocaine craving is associated with electrophysiological brain responses to cocaine-related stimuli. Addiction Biology Sep 2008;13:386-92.

[17] Herrmann MJ, Weijers HG, Wiesbeck GA, Boning J, Fallgatter AJ. Alcohol cuereactivity in heavy and light social drinkers as revealed by event-related potentials. Alcohol and Alcoholism Nov-Dec 2001;36:588-93.

[18] McDonough BE, Warren CA. Effects of 12-h tobacco deprivation on event-related potentials elicited by visual smoking cues. Psychopharmacology Mar 2001;154: 282-91.

[19] Garner DM. Eating Disorder Inventory-2 manual. Odessa, Florida: Psychological Assessment Resources; 1991.

[20] Andrist LC. Media images, body dissatisfaction, and disordered eating in adolescent women. MCN The American journal of maternal child nursing Mar-Apr 2003;28: 119-23.

[21] Dunning JP, Parvaz MA, Hajcak G, Maloney T, Alia-Klein N, Woicik PA et al. Motivated attention to cocaine and emotional cues in abstinent and current cocaine users-an ERP study. European Journal of Neuroscience May 2011;33:1716-23. 